\title{
A Case Report on Severe Hypothyroidism Associated with Complete Bilateral Ptosis: A Rare Presentation
}

\author{
Deepak Jain, H.K. Aggarwal, Shaveta Dahiya \\ Department of Medicine, Pandit Bhagwat Dayal Sharma University of Health Sciences, Rohtak, Haryana, India
}

\begin{abstract}
Thyroid hormones exert a major control over many vital functions of the human body and thus have an important role in maintaining homeostasis. Therefore, the hypothyroid state is associated with a wide spectrum of symptoms affecting almost all bodily functions. Among the major organs affected, nervous system and body metabolism are severely affected. Facial appearance, including ocular changes, is a characteristic of the hypothyroid state. The most prominent ocular features of hypothyroidism include loss of eyelashes and eyebrows, especially on the temporal side, puffiness of the eyelids, ocular irritation and ocular protrusion. Mild drooping of the eyelids is also seen in some patients due to loss of sympathetic tone; however the occurrence of complete bilateral ptosis is rare in the setting of hypothyroidism. Our patient, who presented with sudden onset bilateral ptosis, had no manifestations of diseases involving the ocular structure, cranial nerves or central nervous system primarily; the cause may be attributed to severe hypothyroidism considering the dramatic response to thyroxine replacement therapy.
\end{abstract}

Key words: hypothyroidism, thyroxine, ptosis, myokymia

\section{INTRODUCTION}

Thyroid hormones are major regulators of a number of vital organ systems. Therefore, it is obvious that in a state of depressed hormone production, all the body's major organs are affected. As signs and symptoms develop gradually, many patients seek medical attention many months or years after the disease onset. Hypothyroidism has a higher prevalence in the female population because of its frequent association with autoimmunity. Initial symptoms of disease may be subtle, such as dry coarse skin and cold intolerance that may be ignored before the appearance of symptoms of major system involvement.

Among its varied presentations, involvement of the central nervous system is commonly encountered in hypothyroidism. It can affect both central and peripheral nervous systems at multiple levels along with severe muscular disorders. ${ }^{1}$ Patients may present with fatigue, psychomotor slowing and sometimes with severe intellectual disturbances such as dementia. Proximal myopathy is seen in around $25 \%$ of patients. ${ }^{2}$ Weakness is usually mild and is slowly progressive over months to years. Myxedema is present in around one third of hypothyroid patients. Although ocular symptoms are also present in many patients, manifesting as periorbital puffiness and drooping of eyelids, presenting exclusively as complete ptosis is rare. We report the case of a female who presented with long standing features of

eISSN 2308-118x

Printed in the Philippines

Copyright $(2016$ by the JAFES

Received: March 18, 2016. Accepted: May 11, 2016.

http://dx.doi.org/10.15605/jafes.031.01.12 hypothyroidism with proximal myopathy with sudden onset changes in consciousness levels and bilateral ptosis.

\section{CASE REPORT}

A 55-year-old housewife presented to the emergency department with complaints of excessive drowsiness, decreased oral intake and complete inability to open her eyes for 3 days. In the last three years, she also had difficulty getting up from the squatting position, combing her hair and dressing. These difficulties were gradually progressive with no diurnal variations or periodic fluctuations. She complained of increased sensitivity to cold, dryness of skin, increased hair loss, hoarseness of voice and easy fatigability. She had no history of intake of any prescribed or over-the-counter medications. She also had no history or family history of any major illnesses.

Her detailed physical examination revealed periorbital puffiness and dry, coarse skin with a lemon-yellow tinge. She suffered from extreme psychomotor retardation and only responded to simple motor commands. Complete bilateral ptosis was present although cranial nerve function examination was normal with no facial abnormality. External ocular movements were normal in all directions. No tremors or fasciculations were elicited. Pupillary reaction and pupil size were normal bilaterally. Muscle bulk of the upper and lower limbs was normal. Power in the proximal muscle groups in both upper and

Corresponding author: Deepak Jain, $M D$

Department of Medicine

Pandit Bhagwat Dayal Sharma University of Health Sciences

Rohtak-124001 (Haryana) India

E-mail: jaindeepakdr@gmail.com 
lower limbs was decreased (3/5), however it was normal in the distal group of muscles (5/5). Deep tendon reflexes although present, showed delay in relaxation phase. There was no sensory deficit in any limb. Examination of the pulmonary, cardiovascular and abdominal systems was unremarkable and did not reveal any abnormality.

Her baseline investigations revealed hemoglobin level of 11 $\mathrm{g} / \mathrm{dl}$ with normocytic, normochromic picture on peripheral blood smear with normal serum levels of ferritin, vitamin B12 and folic acid. Renal and liver function analyses along with serum electrolytes were within normal limits. Blood sugar levels and $\mathrm{HbA} 1 \mathrm{c}$ were also normal. Lipid profile was deranged with increased serum triglyceride and cholesterol levels. Further investigations revealed a state of primary hypothyroidism (Table 1). Her CPK levels were raised to $410 \mathrm{U} / \mathrm{L}$ (60-174 U/L). Serum cortisol and Serum ACTH levels were normal.

\section{Table 1. Biochemical parameters of the case}

\begin{tabular}{lll}
\hline Laboratory parameter & Value & Normal range \\
\hline Free T3 & $1.43 \mathrm{pg} / \mathrm{ml}$ & $2.3-4.2 \mathrm{pg} / \mathrm{ml}$ \\
Free T4 & $0.046 \mathrm{ng} / \mathrm{ml}$ & $0.8-1.8 \mathrm{ng} / \mathrm{L}$ \\
TSH & $>100 \mu / \mathrm{U} / \mathrm{ml}$ & $0.5-5 \mu / \mathrm{l} / \mathrm{ml}$ \\
Anti TPO Antibodies & $1160 \mathrm{U} / \mathrm{ml}$ & $<60 \mathrm{Ul} / \mathrm{ml}$ \\
Total CPK & $410 \mathrm{U} / \mathrm{L}$ & $60-174 \mathrm{U} / \mathrm{L}$ \\
Serum Cortisol (7 a.m.) & $12 \mu \mathrm{g} / \mathrm{dl}$ & $7-28 \mu \mathrm{dl}$ \\
Serum ACTH & $8 \mathrm{pg} / \mathrm{ml}$ & $5-27 \mathrm{pg} / \mathrm{ml}$ \\
\hline
\end{tabular}

Electromyographic study revealed myopathic pattern of weakness in the proximal muscle groups of both upper and lower limbs. Repeated nerve stimulation for ocular muscles was negative for myasthenia gravis. Acetylcholine receptor and anti-skeletal antibody levels were not elevated. Cranial MRI and abdominal ultrasound were normal. Contrast-enhanced computed tomography of the chest ruled out any evidence of thymoma. Ultrasound of the thyroid gland revealed small lobes and isthmus with normal blood flow on color doppler. Echocardiography showed grade I diastolic dysfunction with normal left ventricular ejection fraction. Detailed ophthalmic examination ruled out any local, infectious, inflammatory or aponeurotic causes of ptosis.

The patient was started on $100 \mu \mathrm{g}(1.6 \mu \mathrm{g} / \mathrm{Kg}$ body weight) of thyroxine daily. After one week of treatment, the patient dramatically improved, with complete recovery of ptosis and significant improvement in her consciousness level. Her muscle power also improved, although some residual weakness was still persistent. The patient was discharged after 2 weeks of hospitalization on the same treatment, and was advised regular monthly follow up. Her thyroid function tests were repeated every 2 months with necessary titration of the dose until her free T4 and TSH levels were restored to normal range. Her sense of well-being improved gradually, with definitive improvement in muscle power. After 6 months of followup, all her symptoms pertaining to hypothyroidism had resolved and she had no active complaint.

\section{DISCUSSION}

Hypothyroidism is one of the most common endocrine disorders, more frequently affecting females. About 42 million people in India suffer from thyroid disease and iodine deficiency has largely been implicated as the leading cause of hypothyroidism. ${ }^{3}$ Among these patients, autoimmune thyroiditis was demonstrable in $7.5 \%$ of subjects. ${ }^{4}$ Hashimoto's thyroiditis is the most common form of autoimmune thyroid disease in areas of iodine deficiency, caused by $\mathrm{T}$-cell mediated disease with a strong genetic component. ${ }^{5}$ It is usually diagnosed by demonstration of anti-thyroid antibodies and findings of thyroiditis on ultrasonography. Secondary hypothyroidism from pituitary or hypothalamic dysfunction is usually rare.

Thyroid hormones exert control over basic metabolic functions of the body, therefore its deficiency is expected to produce a wide array of symptoms. It covers a wide spectrum of clinical and biochemical diseases, from clinically inapparent disease to myxoedema coma. ${ }^{6}$ Hypothyroidism emerges insidiously and is nonspecific. The clinical features may be atypical, and the diagnosis may be missed easily particularly in the elderly. ${ }^{7}$

Without regard to the cause of hypothyroidism, neuromuscular and musculoskeletal manifestations are present in many of these patients. ${ }^{8}$ The hypothyroid state severely impairs protein and carbohydrate metabolism affecting many organ systems, especially the muscles manifesting as pain with muscle exertion. A shift in distribution of muscle fiber types is also observed as shift from fast twitch fibers to slow twitch fibers which causes slowed muscle contraction and relaxation known as "hypothyroid myopathy." A reduction in muscle mitochondrial oxidative capacity and beta-adrenergic receptors, as well as the induction of an insulin-resistant state, may result in these changes. Hypothyroid myopathy typically manifests as polymyositis-like myopathy with proximal muscle weakness and an increased creatine kinase level. ${ }^{9}$ Typical facial features and ocular changes
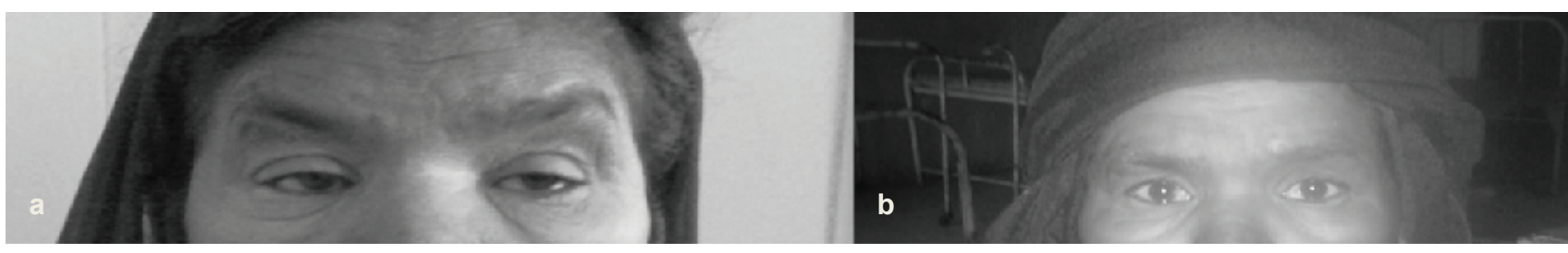

Figure 1a. Showing ptosis in patient on day 4 of admission. Figure 1b. Showing complete recovery on day 7 of admission. 
are characteristic of hypothyroid state manifesting with loss of eyelashes and eyebrows especially on the temporal side, puffiness of eyelids, ocular irritation and ocular protrusion. These are largely attributed to accumulation of glycosaminoglycans and cross reactivity of autoantibodies with orbital and dermal fibroblasts.

The occurrence of complete bilateral ptosis is very rare in the setting of hypothyroidism, although mild drooping of eyelids may be present in some patients due to loss of sympathetic tone. Since our patient, who presented with sudden onset bilateral ptosis had no manifestations of disease involving the eye, cranial nerves or central nervous system primarily; the cause may be attributed to severe hypothyroidism, particularly the ptosis responded dramatically to thyroxine replacement therapy. Various mechanisms may be postulated, although the exact cause is still unknown.

Myokymic discharges may be the etiology behind this rare manifestation..$^{10}$ An autoimmune basis due to antibody interference with the function of potassium channels, leading to increased nerve terminal excitability, is a documented cause of myokymia. ${ }^{11}$ Another hypothesized mechanism is focal demyelination involving isolated terminal branches to the orbicularis oculi followed by multiple grouped discharges on demyelinated axon terminals leading to myokymia and persistent ptosis. Lee et al. reported a similar case of ptosis due to hypothyroidism which was secondary to Sheehan's syndrome. ${ }^{12}$ Cho et al. described a case of pituitary apoplexy with Sheehan's syndrome and isolated third cranial nerve palsy. In their case, direct mechanical compression of the third cranial nerve or the vascular supply to the nerve resulted in a sudden onset of isolated third cranial nerve palsy. The patient's ptosis was improved by surgical decompression. ${ }^{13}$ Proximal myopathy and severe bilateral ptosis due to hypothyroidism were also reported by Green $\mathrm{PH}$ in a 28 year-old female. ${ }^{14}$ Apart from these, there have been few case reports of patients of myasthenic syndromes who responded completely with thyroxine treatment alone. ${ }^{15}$

\section{CONCLUSION}

In this paper, we intend to highlight the unusual presentation of hypothyroid state in the form of sudden onset bilateral ptosis with features of proximal myopathy and no other identifiable etiology for this presentation. This is further supported by the fact that the patient completely recovered with thyroxine replacement therapy alone.

\section{Ethical Consideration}

Patient consent form has been procured prior to the case report study.

\section{Statement of Authorship}

All authors have given approval to the final version submitted.

\section{Conflict of Interest}

All the authors have declared no conflict of interest to the work carried out in this paper.

\section{Funding Source}

This study has not been funded by any agency or grant.

\section{References}

1. Duyff RF, Van den Bosch J, Laman DM, Van Loon BJ, Linssen WH. Neuromuscular findings in thyroid dysfunction: A prospective clinical and electrodiagnostic study. J Neurol Neurosurg Psychiatry. 2000;68(6):750-5. PMID: 10811699.

2. Rao S, Katiyar B, Nair K, Misra S. Neuromuscular status in hypothyroidism. Acta Neurol Scand. 1980;61(3):167-77. http://dx.doi. org/10.1111/j.1600-0404.1980.tb01479.x.

3. Unnikrishnan AG, Menon UV. Thyroid disorders in India: An epidemiological perspective. Indian J Endocrinol Metab 2011;15(2): 78-81. http://dx.doi.org/10.4103/2230-8210.83329.

4. Woeber KA. Update on the management of hyperthyroidism and hypothyroidism. Arch Intern Med. 2000;160(8):1067-71. http://dx.doi. org/10.1001/archinte.160.8.1067.

5. Singh SK, Singh KK, Sahay RK. Images in medicine: Hashimoto's thyroiditis with orbitopathy and dermopathy. J Postgrad Med. 2000;46(4):286-7. PMID: 11435659.

6. Jayakumar RV. Hypothyroidism. J Indian Med Assoc. 2006;104(10): 557-60,562. PMID: 17380819.

7. Hallengren B. Hypothyroidism-clinical findings, diagnosis, therapy. Thyroid tests should be performed on broad indications. Lakartidningen 1998;95(38):4091-6. PMID: 9772803.

8. Barohn R. Metabolic myopathies. In: Cecil RL, Goldman L, eds. Textbook of Medicine. 21 ${ }^{\text {st }}$ ed. Orlando, Fla: Harcourt Health Sciences; 2000.

9. Miyake I, Hiromatsu Y. Hypothyroid myopathy. Ryoikibetsu Shokogun Shirizu. 2001;(36):294-7. PMID: 11596395.

10. Lo YL, Ho SC, Koh LK, Kooh DH. EMG myokymia as a cause of ptosis in hypothyroidism. Eur J Neurol. 2003;10(1):87-90. http://dx.doi.org/10.1046/j.1468-1331.2003.00544.x.

11. Newsom-Davis J, Mills KR. Immunological associations of acquired neuromyotonia (Isaac's syndrome). Report of 5 cases and literature review. Brain. 1993;116(2):453-69. http://dx.doi.org/10.1093/brain/116.2.453.

12. Lee YS, Moon SS. A case of Sheehan's syndrome that manifested as bilateral ptosis. J Korean Med Sci. 2011;26(4):580-2. http://dx.doi.org/ 10.3346/jkms.2011.26.4.580.

13. Cho WJ, Joo SP, Kim TS, Seo BR. Pituitary apoplexy presenting as isolated third cranial nerve palsy with ptosis: Two case reports. J Korean Neurosurg Soc 2009;45:118-121.

14. Green PH. Bilateral ptosis and proximal myopathy - muscular manifestations of hypothyroidism. Aust N Z J Med. 1977;7(4):418-9. http://dx.doi.org/10.1111/j.1445-5994.1977.tb04408.x.

15. Forbes H, Norris JR. Neuromuscular transmission in thyroid disease. Ann Intern Med. 1966;64(1):81-6. http://dx.doi.org/10.7326/00034819-64-1-81.

Authors are required to accomplish, sign and submit scanned copies of the JAFES Author Form consisting of: (1) the Authorship Certification that the manuscript has been read and approved by all authors, and that the requirements for authorship have been met by each author, (2) the Author Declaration that the article represents original material that is not being considered for publication or has not been published or accepted for publication elsewhere, (3) the Statement of Copyright Transfer[accepted manuscripts become the permanent property of the JAFES and are licensed with an Attribution-Share Alike-Non-Commercial Creative Commons License. Articles may be shared and adapted for non-commercial purposes as long as they are properly cited], (4) the Statement of Disclosure that there are no financial or other relationships that might lead to a conflict of interest. For Original Articles involving human participants, authors are required to submit a scanned copy of the Ethics Review Approval of their research. For manuscripts reporting data from studies involving animals, authors are required to submit a scanned copy of the Institutional Animal Care and Use Committee approval. For Case Reports or Series, and Images in Endocrinology, consent forms, are required for the publication of information about patients; otherwise, authors declared that all means have been exhausted for securing such consent. Articles and any other material published in the JAFES represent the work of the author(s) and should not be construed to reflect the opinions of the Editors or the Publisher. 